\title{
EchoGéo
}

$17 \mid 2011$

Activités extractives

\section{Croissance économique des pays émergents et géographie mondiale des pierres précieuses}

\section{Remy Canavesio}

\section{OpenEdition}

1 Journals

Édition électronique

URL : https://journals.openedition.org/echogeo/12523

DOI : $10.4000 /$ echogeo. 12523

ISSN : 1963-1197

Éditeur

Pôle de recherche pour l'organisation et la diffusion de l'information géographique (CNRS UMR 8586)

Référence électronique

Remy Canavesio, «Croissance économique des pays émergents et géographie mondiale des pierres précieuses », EchoGéo [En ligne], 17 | 2011, mis en ligne le 27 septembre 2011, consulté le 10 août 2021. URL : http://journals.openedition.org/echogeo/12523; DOl : https://doi.org/10.4000/echogeo. 12523

Ce document a été généré automatiquement le 10 août 2021.

EchoGéo est mis à disposition selon les termes de la licence Creative Commons Attribution - Pas d'Utilisation Commerciale - Pas de Modification 4.0 International (CC BY-NC-ND) 


\title{
Croissance économique des pays émergents et géographie mondiale des pierres précieuses
}

\author{
Remy Canavesio
}

\section{Introduction}

Bien que les espaces producteurs de gemmes ${ }^{1}$ aient fait l'objet de nombreuses études, leur connaissance reste très lacunaire car elle souffre d'un déséquilibre majeur au profit des seules régions diamantifères, notamment africaines. Les travaux produits par la société civile autour de la dénonciation des diamants du sang (rapports des ONG Global Witness et Partenariat Afrique Canada notamment) et les nombreux ouvrages analysant les mutations récentes de la filière (Misser et Valle, 1997; Brunet, 2003 ; De Geloes D'Elsloo et al., 2004 ; Smillie, 2010...) ont largement contribué à cette inégalité de traitement. La géographie mondiale des autres pierres précieuses et semi-précieuses (saphirs, rubis, émeraudes, béryls, grenats...) est en revanche très peu étudiée.

2 Cette géographie forme un ensemble hétérogène avec des lieux d'extraction, des filières et des marchés spécifiques à chaque gemme. Bien qu'il fasse appel à de multiples exemples - et notamment au diamant -, cet article propose de porter une attention particulière aux cas des saphirs et des rubis. Alors que la croissance économique des pays émergents transforme l'économie mondiale, on peut s'interroger sur les conséquences de ce processus sur la géographie mondiale des pierres précieuses. Cette question peut être abordée sous l'angle de la demande, en observant l'effet de l'enrichissement de la population sur la consommation de pierres précieuses. Elle peut également être analysée par le prisme de l'offre, en mettant en évidence les liens mal connus existant entre l'extraction des pierres précieuses et le contexte socioéconomique des pays producteurs. Cette approche essentiellement économique est justifiée par le fait que, contrairement à beaucoup d'autres ressources à forte valeur avec lesquelles ces gemmes semblent pouvoir être comparées (or, coltan, diamant, etc.), 
l'exploitation des saphirs et des rubis est très peu affectée par les évolutions de la législation minière car c'est une activité essentiellement informelle. De plus, elle n'est pas l'objet d'enjeux géopolitiques internationaux dans la mesure où ces pierres sont dépourvues d'intérêt stratégique. On s'apercevra donc que ces dynamiques économiques et sociales permettent d'appréhender l'essentiel des évolutions actuelles d'un grand nombre de régions productrices d'Asie méridionale et d'Afrique subsaharienne.

3 Pour commencer, ce travail dressera les contours du marché des gemmes dans les pays à forte croissance économique. Cela devrait permettre d'évaluer si les produits d'ornementation utilisés en joaillerie sont soumis à une augmentation de la demande également répartie de la part des populations qui accèdent à la classe moyenne et supérieure dans les pays en développement. On verra ensuite si l'évolution des contextes socio-économiques est également capable d'affecter l'offre, et si les différentes évolutions constatées au sein des pays du Sud conduisent à une redistribution de la géographie mondiale de l'extraction des saphirs et des rubis.

4 Apporter des réponses à ces questions impose de se confronter à la délicate question des sources. Si les grands traits du commerce diamantaire mondial peuvent être dressés assez aisément à partir des statistiques des acteurs officiels (groupes miniers notamment), l'étude des autres gemmes est très complexe en raison du nombre élevé d'acteurs, du caractère souvent informel de ceux-ci, et en raison des nombreuses fraudes qui caractérisent ce commerce. Trouver des chiffres fiables permettant de mesurer les tendances est une véritable gageure et les analyses présentées ici sont avant tout le fruit des entretiens réalisés avec les collecteurs de pierres précieuses rencontrés dans les régions minières de Madagascar ${ }^{2}$, en Thaïlande ainsi que par le recoupement d'informations glanées sur Internet ${ }^{3}$.

\section{La croissance des pays émergents et ses conséquences sur la demande en produits de joaillerie}

\section{Le rôle de la matrice historique et spatiale ${ }^{4}$ dans le comportement des consommateurs}

5 Le marché des produits de joaillerie est singulier à bien des égards. Cela est particulièrement marqué du point de vue des gemmes, qui semblent exercer une fascination très variable d'un pays à l'autre (Brunet, 2003; Canavesio, 2010). Le comportement des consommateurs vis-à-vis de ces produits de luxe n'est pas égal en tout point du monde. Il est très fortement influencé par les héritages historiques et spatiaux. Si le diamant se présente comme un produit relativement standardisé et "déterritorialisé », de nombreuses pierres précieuses (émeraudes saphirs, rubis) et pierres fines peuvent être comparées à des produits « de terroir» (Canavesio, 2010). Cette particularité joue un rôle important dans l'attitude des consommateurs. Cette dernière est en grande partie l'héritage d'une tradition qui se perpétue, en évoluant toutefois plus ou moins rapidement au gré des modes et des opérations de marketing. Les choix des consommateurs actuels sont donc largement dépendants des comportements passés, ceux-ci étant parfois dictés par la présence de gisements de première importance plus ou moins proches des régions de consommation. En effet, même si les gemmes ont des caractéristiques qui permettaient leur commercialisation 
sur de longues distances avec des moyens de transports rudimentaires (Giuliani, et al., 2000), on retrouve fréquemment les traces de pratiques séculaires influencées par la proximité et l'accessibilité à ces ressources ${ }^{5}$.

Ce lien qui se maintient souvent entre l'existence d'espaces d'extraction de gemmes anciens et le comportement actuel des consommateurs est à l'origine d'une bonne part des singularités actuelles des principaux marchés de la joaillerie mondiale. L'occident, largement dépourvu en gisements ${ }^{6}$, est longtemps resté en marge de ces routes commerciales anciennes. La "consommation» de gemmes n'a jamais imprégné la culture européenne comme celle de certaines régions d'Asie méridionale. La colonisation européenne et l'enrichissement des occidentaux marquèrent un tournant décisif. La démocratisation de l'intérêt pour les gemmes, jusque-là réservé aux plus puissants, est intervenue au même moment que la découverte de plusieurs gisements majeurs de diamants dans les possessions coloniales. Le système commercial mondialisé qui s'est mis en place entre les colonies sud-américaines, africaines et l'Europe fut ainsi le point de départ de la domination du diamant sur le marché occidental. Cette prédominance se renforça sans cesse au $\mathrm{XX}^{\mathrm{e}}$ siècle en Europe et en Amérique du Nord, sous l'effet des stratégies des acteurs d'une filière essentiellement formelle et bien structurée (situation de monopole de la société De Beers). Le marché américain qui représentait $31 \%$ du marché mondial de la joaillerie en 2005 (KPMG, 2006), fut une cible privilégiée du marketing diamantaire. Les diamants représentent à l'heure actuelle près de $95 \%$ des ventes de gemmes aux États-Unis (ibidem). Avec quelques variations d'un pays à l'autre ${ }^{7}$, le consommateur européen se caractérise par un comportement équivalent, même si la prééminence du diamant y est légèrement plus modeste (Brunet, 2003). Les évolutions et les temporalités du marché japonais sont assez semblables. En effet, la culture nippone accorde un intérêt très limité aux différentes gemmes mais - du fait du pouvoir d'achat élevée de la population - le Japon est devenu un des principaux marchés pour plusieurs produits de joaillerie. Le diamant domine très nettement les autres gemmes avec près de $90 \%$ des ventes $^{8}$ alors que, plus généralement, l'influence occidentale est prépondérante sur le secteur du luxe nippon (Siboni, 2007).

$7 \quad$ Ayant évolué dans des contextes différents, la plupart des pays émergents ont conservé de fortes singularités. Celles-ci s'avèrent d'autant plus intéressantes à étudier que ces marchés sont en passe de porter l'essentiel de la croissance mondiale de la consommation de gemmes. Le cas des puissances asiatiques est particulièrement révélateur.

8 L'Asie méridionale est depuis longtemps le centre de gravité de l'extraction, du façonnage et du commerce des gemmes (hors diamant). Le sous-continent indien et ses marges fournissent depuis plusieurs millénaires une très grande diversité de pierres précieuses et de pierres fines (saphirs, rubis, émeraudes, diamants, jade, spinelles...). Les montagnes du Pakistan et d'Afghanistan, le Sud de l'Inde, le Sri Lanka et la Birmanie sont connus depuis des millénaires pour leurs gisements (Hughes, 1997b). Dans ce vaste ensemble (illustration 1) peuplé de près d'un milliard et demi d'individus, les gemmes font partie intégrante de la culture. "Precious gems and jewellery are a part and parcels of Indian traditions and customs.» (India Law Offices, 2008, p. 1). Elles continuent de jouer un rôle majeur dans les grandes étapes de la vie car elles sont souvent considérées comme étant dotées de "pouvoirs" surnaturels". Leur diversité est connue et appréciée. Cela explique que le diamant ne jouisse pas d'une suprématie comparable à 
celle dont il profite sur les marchés occidentaux. S'il est quasiment impossible de chiffrer ${ }^{10}$ avec précision la part de marché de chacune des gemmes dans ces pays, on peut néanmoins affirmer que les saphirs, rubis, émeraudes et les nombreuses pierres semi-précieuses occupent une part importante du marché global des gemmes en Asie méridionale.

9 L'Asie orientale est devenue le principal moteur mondial du marché du luxe dans la dernière décennie (Siboni, 2007). Elle est marquée par un spectre de consommation des gemmes bien différent. En Chine notamment, la joaillerie est traditionnellement dominée par le travail des métaux précieux (or, argent), qui sont également très largement considérés du point de vue de leur capacité à fournir une forme d'épargne ${ }^{11}$ (Song, 2004). Les gemmes ne font pas partie intégrante de la culture et seul le cas du jade mérite d'être distingué. En effet, cette pierre qui est largement boudée par les consommateurs occidentaux, est l'objet d'un engouement ancien et croissant sur ce marché (Tsai, 2008). Ce dernier est alimenté depuis des siècles en jade provenant de gisements de la frontière sino-birmane ${ }^{12}$. Là encore, le rôle de la proximité des espaces extractifs sur le comportement des consommateurs semble avoir été déterminant.

Par ailleurs, et sans entrer dans le détail de chacune de leurs singularités, on notera que d'autres grands marchés dynamiques (pays du Golfe persique, Brésil, Russie....) sont également marqués par ce type d'héritages culturels et spatiaux. Les collecteurs de gemmes sri lankais rencontrés à Madagascar affirment par exemple que les riches consommateurs des pétro-monarchies du golfe persique montrent une attirance exacerbée pour les gemmes de couleur verte (couleur de l'islam) ainsi que pour certaines pierres largement ignorées des occidentaux (lapis-lazuli, chrysobéryls, alexandrites...).

\section{Un potentiel de croissance immense mais inégal dans les pays émergents}

11 Les marchés occidentaux et japonais sont désormais matures et leur progression devrait rester modeste, à l'image d'une croissance économique molle et incertaine. Les pays émergents représentent en revanche un potentiel de croissance majeur pour le secteur de la joaillerie, même si cette dernière pâtit de sa désorganisation au profit des autres secteurs du luxe (Song, 2004; KPMG, 2006). Les perspectives sont particulièrement intéressantes en Inde et en Chine : « ...jewellery is traditionally a luxury item of consumption, and demand for jewellery is highly elastic to income. As China's per capita disposable income rises, jewellery, after real estates and automobiles, has emerged as the third hottest item of consumption in recent times " (Embassy of India, 2004, p. 4).

En 2005, la Chine et l'Inde représentaient chacune près de $10 \%$ du marché mondial de la joaillerie (KPMG, 2006). L'ensemble du secteur profite de ce dynamisme, avec une croissance moyenne de près de $5 \%$ par an dans la dernière décennie. L'Inde est le premier consommateur d'or (près de $20 \%$ de la consommation mondiale) et les gemmes sont l'objet d'un engouement sans précédent. Selon certains acteurs de la filière, les marchés chinois et indiens pourraient même égaler le marché américain dès 2015 (KPMG, 2006).

13 En termes qualitatifs, l'évolution sera probablement plus contrastée même si de nouveaux comportements peuvent apparaitre et transformer les pratiques héritées. Pour le secteur de la joaillerie, le marché nippon semble préfigurer l'évolution de la 
Chine. En effet, le marché chinois offre un espace relativement vierge dans lequel le marketing diamantaire peut s'exprimer sans rencontrer d'entraves culturelles majeures. En dehors de son attirance pour le jade, le consommateur chinois se tourne de plus en plus massivement vers le diamant qui, à l'inverse des autres gemmes, bénéficie d'une promotion active menée par des acteurs institutionnalisés et puissants. "Every year, about 18-22 million people in China get married (...). The younger consumer section is also fashion-conscious (...). According to consumer research of Diamond Trading Company (DTC), a subsidiary of the De Beers Group, three out of four brides in Beijing and Shanghai expect their husbands to buy them diamond wedding ring. Wedding rings account for one-third of all diamond sales in China » (Embassy of India in Beijing, 2004, p. 5).

Dans ce domaine, l'entrée de la De Beers sur le marché chinois en 1994 représenta une étape décisive. Par ailleurs, contrairement au « monde indien », dans lequel les gemmes sont porteuses de valeurs multiples (divines, sociales, thérapeutiques, astrologiques...), le consommateur chinois est surtout sensible à la mode et à la signification de la joaillerie en terme de statut social (Song, 2004; Siboni, 2007). Cet environnement est donc extrêmement favorable au diamant, que ce soit pour des raisons intrinsèques (standardisation du produit) ou de structure de la filière (capacité des acteurs formels à influencer le choix des consommateurs). Les ventes de diamant progressent ${ }^{13}$ d'ailleurs à un rythme plus élevé que celui des autres gemmes ${ }^{14}$ (Pan, 2008), mais également plus vite que la croissance économique globale du pays.

L'Asie méridionale observe également une vive croissance de ce marché, mais le contexte semble moins favorable au diamant. Pour des raisons intrinsèques, de politique et de relation à l'occident $\left(\operatorname{Sen}^{15}, 2007\right)$, la culture du monde indien se manifeste actuellement par de très fortes résistances à l'encontre de l'occidentalisation des comportements. Alors que la région connaît une croissance économique soutenue, on peut supposer que l'attirance millénaire pour les gemmes devrait conduire ce marché à atteindre des proportions exceptionnelles. Il est en revanche peu probable que le diamant parvienne à s'imposer de manière aussi magistrale que sur les marchés occidentaux japonais et chinois. Ainsi, même si les ventes de ce dernier devraient continuer à progresser à un rythme rapide, l'Asie méridionale pourrait surtout devenir le principal marché mondial pour les pierres telles que le rubis ou le saphir, ainsi que pour toute une gamme de pierres semi-précieuses pour lesquelles les indiens ont une attirance millénaire.

En fin de compte, on constate que l'augmentation du niveau de vie dans les pays émergents est un puissant facteur de croissance pour le commerce mondial des gemmes. Si le diamant est particulièrement prisé par l'ensemble des consommateurs, l'Asie méridionale se distingue par un intérêt également très vif pour les autres gemmes et notamment pour les saphirs et les rubis. Il convient maintenant de voir comment le marché parvient à répondre à cette demande nouvelle. On verra que contrairement à la réaction attendue, qui voudrait que l'augmentation de la demande stimule l'offre locale, l'enrichissement de la population peut également conduire à une réduction de la production dans certains espaces extractifs d'Asie du Sud. 


\section{Quand l'enrichissement transforme la géographie de l'offre}

\section{Entre exploitations formelles mécanisées et mines artisanales}

17 Avec certains métaux précieux tels que l'or ou le coltan, les gemmes sont une des rares ressources minières dont l'extraction soit encore en grande partie le fait d'activités artisanales informelles. Pour les saphirs et les rubis, la mine artisanale représente même probablement plus de $80 \%$ de la production mondiale ${ }^{16}$. La très grande valeur de cette ressource (par rapport à son poids et à son volume) fait que son exploitation non mécanisée peut demeurer rentable jusqu'à des concentrations relativement faibles pour certaines populations particulièrement déshéritées.

Ainsi, alors que l'extraction artisanale a quasiment disparu de la plupart des gisements de produits miniers à faible valeur relative, plusieurs centaines de milliers de mineurs ${ }^{17}$ s'affèrent dans des gisements de pierres précieuses et de pierres fines des pays du Sud. L'activité minière peut y devenir le moteur économique de régions entières en dépit de chiffres d'affaires souvent peu élevés en comparaison de certaines productions minières formelles (Canavesio, 2010). En effet, lorsqu'elle est artisanale, l'extraction des gemmes requiert une quantité importante de main-d'œuvre. En Afrique, en Amérique $\mathrm{du}$ Sud et en Asie méridionale, plusieurs régions sont ainsi très profondément marquées par ces activités extractives depuis des dizaines d'années, voire des siècles. Sur le continent africain, plusieurs espaces diamantifères célèbres sont ainsi reconnus : le Lunda Norte en Angola, le Kasaï au Sud de la République Démocratique du Congo, la Sierra Leone et le Sud de la République Centrafricaine notamment. En Amérique du Sud, les émeraudes et certaines pierres fines (améthystes, tourmalines) jouent parfois un rôle comparable dans le département du Boyaca en Colombie ou dans l'Etat du Minas Gerais au Brésil par exemple. Enfin, en Asie méridionale, plusieurs régions (illustration 1) connaissent une activité extractive artisanale, parfois depuis des millénaires (Hughes, 1997b). Le Sud du Sri Lanka, la frontière thaïlando-cambodgienne et la région de Mogok en Birmanie sont ainsi de très hauts lieux de l'extraction des saphirs et des rubis, autour desquels se sont structurées la plupart des filières internationales contemporaines. 
Illustration 1 - Espaces d'extraction et principales routes commerciales des gemmes en Asie méridionale

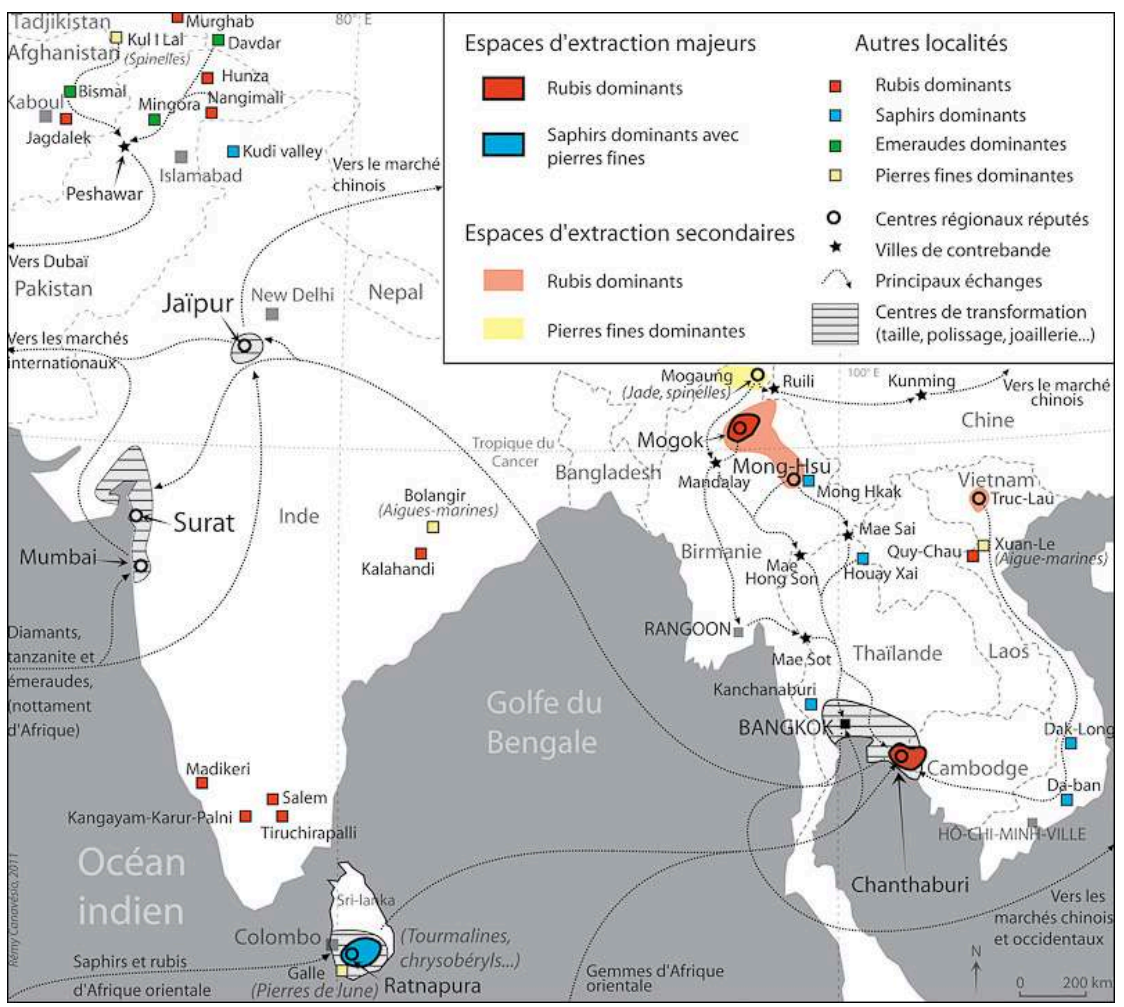

Auteur : Remy Canavesio, 2010, modifié.

Dans chacun de ces cas, le haut niveau d'artisanat dans les méthodes d'extraction des gemmes est conditionné par deux facteurs principaux. L'existence de gisements à la rentabilité nulle ou aléatoire pour les exploitations fortement mécanisées d'une part, et la permanence de crises socioéconomiques produisant une précarité suffisante pour que les populations soient contraintes à ce type d'activité à la fois dangereuses et faiblement rémunératrices ${ }^{18}$, d'autre part. Dans le cas contraire, la mine artisanale est soit remplacée par des sociétés formelles lourdement mécanisées (cas des gisements à très fort potentiel), soit l'activité minière ne se développe jamais ou périclite en raison du désintérêt des populations locales pour ces activités pénibles et hasardeuses (cas des gisements d'intérêt économique faible dans les pays développés ${ }^{19}$ ). Dans le domaine des gemmes, l'évolution de la géographie des espaces extractifs n'est donc pas le simple produit d'un rapport entre le niveau de l'offre "géologique " (existence, découverte, épuisement des gisements) et celui de la demande. Les évolutions des législations minières interviennent également très peu dans ces dynamiques ${ }^{20}$. En fait, ces changements sont avant tout dictés par les transformations socioéconomiques des pays abritant des gisements. Cela explique que, en plus de stimuler la consommation de produits de joaillerie, l'enrichissement de la population dans certains pays émergents devienne un nouveau facteur d'évolution de la géographie mondiale des exploitations de saphirs et de rubis. En effet, dans la mesure où ces deux gemmes sont particulièrement concernées par les exploitations informelles (Canavesio, 2010), l'activité extractive est davantage liée aux phénomènes sociaux, économiques et politiques qu'aux évolutions actuelles de la législation minière. Néanmoins, cette interaction entre l'exploitation des saphirs et des rubis et la sphère économico- 
politique n'a jamais été aussi complète que dans certains pays diamantifères africains. En effet, si les crises ont soutenu l'activité minière informelle, cette dernière n'a - dans le cas de l'exploitation des saphirs et des rubis - jamais été la source principale de conflits nationaux ou régionaux ${ }^{21}$.

Lorsque les gisements offrent des concentrations importantes et des perspectives d'exploitation à long terme, ils échappent aux méthodes artisanales et informelles et font l'objet d'exploitations lourdement mécanisées très peu dépendantes des conditions socioéconomiques régionales. C'est par exemple le cas des très nombreuses cheminées de kimberlite diamantifères exploitées par les grandes compagnies minières multinationales (De Beers, Alrossa, BHP, Rio Tinto...). Ces exploitations fortement capitalistiques ne produisent pas les mêmes effets sur les espaces extractifs. Si elles dégagent des chiffres d'affaires très nettement supérieurs aux exploitations artisanales, et si elles ont généralement un impact environnemental et paysager également plus important $t^{22}$, elles sont en revanche beaucoup moins structurantes au niveau des systèmes sociaux, économiques et territoriaux des espaces qu'elles investissent. Ces exploitations qui peuvent contribuer massivement au revenu des États (exemple du Botswana avec l'exploitation diamantaire) ne proposent, en retour, que très peu d'emplois (quelques centaines au maximum) et forment souvent des enclaves très peu intégrées dans les sociétés locales.

\section{Transformation de la géographie mondiale des gemmes}

21 En réponse à la hausse rapide de la demande, les différentes filières ont connu des évolutions contrastées. Le milieu du diamant, qui s'est efforcé de mettre fin à ses dérives criminelles ${ }^{23}$ en développant la traçabilité des pierres brutes (processus de Kimberley), a également vu apparaître de nouveaux pays producteurs et de nouveaux acteurs. Alors que le marché était déjà marqué par une offre excédentaire conduisant la De Beers à former des stocks colossaux pour maintenir les prix à un niveau élevé, de nouvelles exploitations formelles importantes ont vu le jour. Le Botswana et l'Australie dans les années 1980, puis le Canada à partir des années 2000, ont ainsi fourni l'essentiel de l'accroissement de la production mondiale au sein de mines géantes appartenant à diverses multinationales (Brunet, 2003). Les exploitations artisanales de diamant (presque exclusivement africaines) ont très peu contribué à cet élan. Au contraire, plusieurs d'entre elles ont connu une baisse de leur production globale (formelle et informelle) dans les deux dernières décennies. Cela peut s'expliquer à la fois par l'épuisement progressif des gisements et par une globale stabilité des prix, qui n'encourage pas la mise en œuvre d'efforts supplémentaires pour exploiter des gisements de plus en plus difficile d'accès. C'est notamment le cas des régions diamantifères centrafricaines et dans une moindre mesure, angolaises, dont une bonne part des gisements de surface a déjà été largement exploitée. Malgré le dynamisme de la demande dans les pays émergents, il est peu probable que la tendance change radicalement dans les décennies à venir. Le marché du diamant devrait continuer de rester bien approvisionné en raison d'un potentiel géologique important dans des gisements à forte rentabilité économique, qu'ils soient avérés (Canada, Sibérie) ou supposés (Arctique européen, Australie...). Par ailleurs, les stocks actuels et la perspective du développement du commerce des diamants « d'occasion » (Brunet, 2003) devraient également permettre de satisfaire durablement la croissance du marché. Il est donc très peu probable que de nouveaux grands espaces extractifs de diamant 
marqués par la mine artisanale ne voient le jour dans les pays du Sud pour répondre à la demande émanant des pays émergents.

Illustration 2 - Évolution de l'Indice de Développement Humain et de la production de saphirs et de rubis dans les principaux pays producteurs depuis 1975

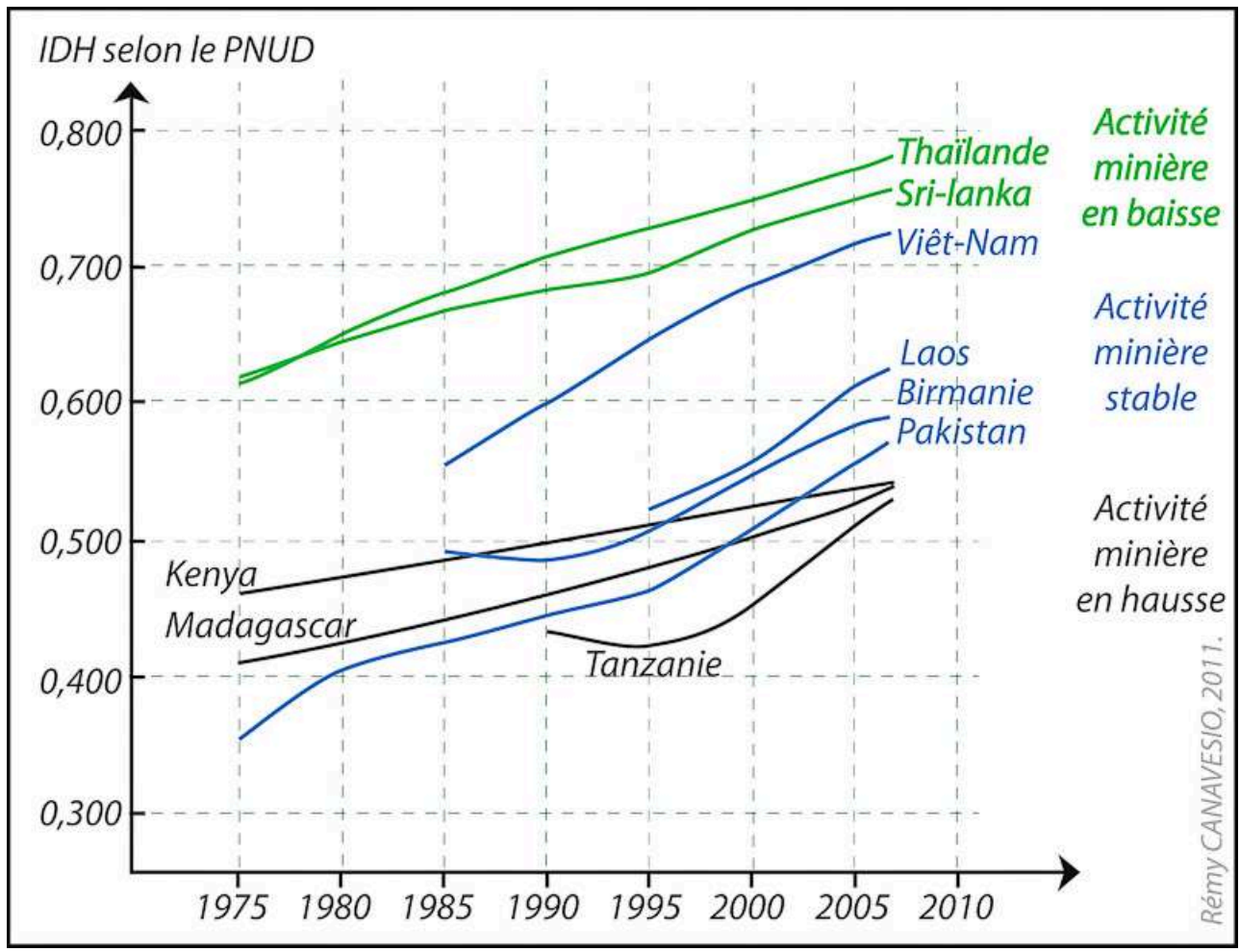

Pour les autres gemmes, et notamment pour les saphirs et les rubis, la situation est bien différente. Les très nombreuses transformations connues par la géographie de ces espaces extractifs dans les vingt dernières années préfigurent probablement l'avenir. Le dynamisme économique actuel de l'Asie méridionale a déjà commencé à produire des changements. Les anciens espaces extractifs de cette région ont de plus en plus de mal à alimenter la croissance du marché mondial, malgré les évolutions des traitements ${ }^{24}$ permettant de rendre commercialisable un nombre grandissant de pierres qui étaient jusque-là inexploitables en joaillerie. L'épuisement progressif des gisements "historiques" ne permet pas d'expliquer l'ensemble du décalage qui se forme entre l'offre et la demande sur le marché de ces gemmes. Alors qu'une classe moyenne se constitue dans cette partie du monde - conduisant à accroître massivement la demande pour ces pierres culturellement très attractives l'enrichissement progressif de l'ensemble de la population commence également à modifier l'offre, en tarissant progressivement le flux de candidats à l'exploitation artisanale des gemmes dans les pays dont la situation économique et sociale est la plus favorable. Ce phénomène est surtout sensible en Thaïlande (et dans une moindre mesure au Sri Lanka) où plusieurs gisements très peu rentables ont été désertés par des mineurs de moins en moins nombreux depuis une quinzaine d'année (Hughes, 1996). En effet, en Asie, parmi les grands pays producteurs de gemmes, la Thaillande et le Sri Lanka sont ceux qui ont atteint le plus haut Indice de Développement Humain (IDH) (illustration 2). Dans ce contexte, en dehors des évolutions techniques proposées par 
l'évolution des traitements, comment le marché parvient-il à répondre à la forte croissance de la demande émanant des pays émergents?

Illustration 3 - Nouveau village de mineurs artisanaux et informels à côté d'Ilakaka à Madagascar

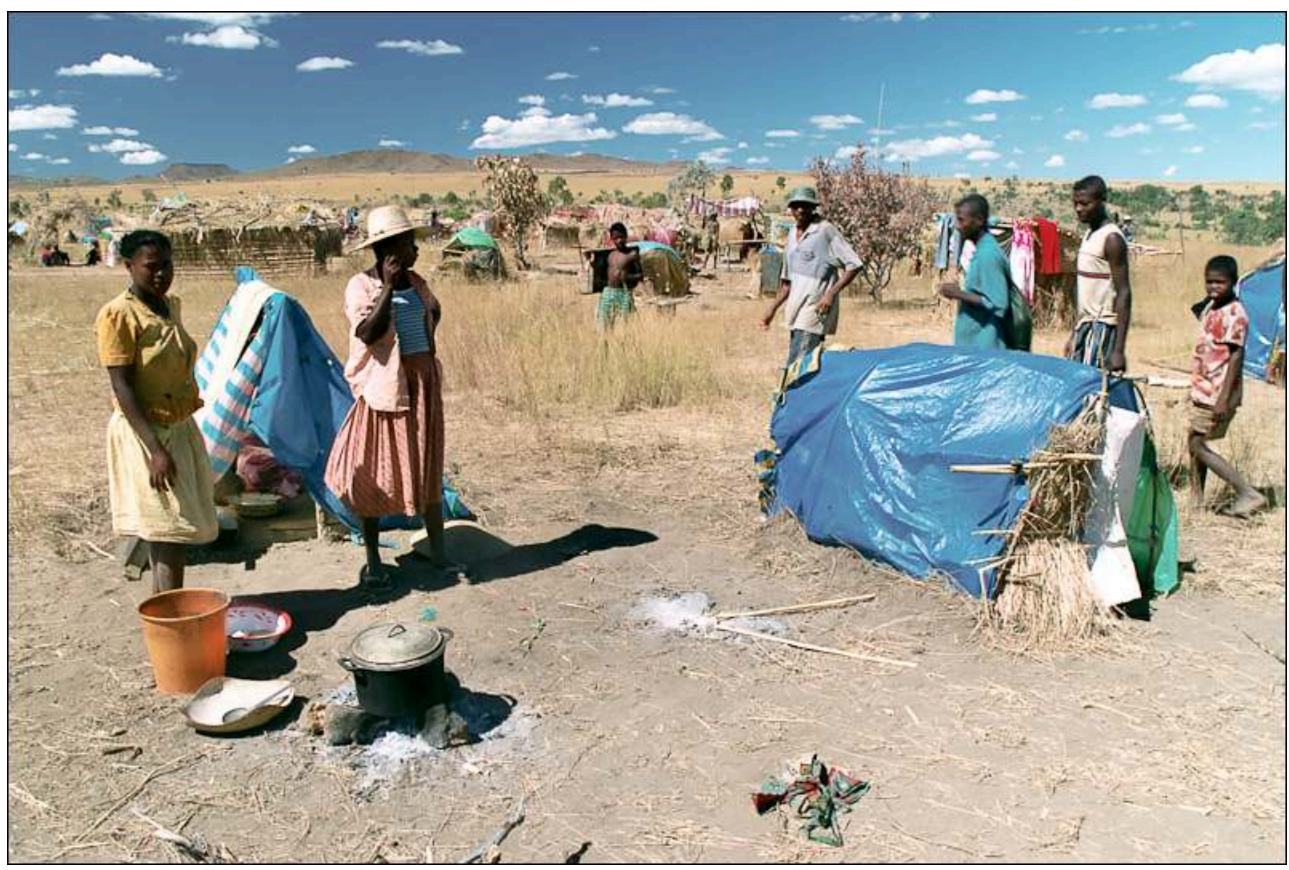

Auteur : Remy Canavesio, avril 2006.

L'Afrique de l'Est semble toute désignée pour prendre le relais de ces espaces extractifs historiques d'Asie méridionale. Cette partie du monde cumule des atouts majeurs pour devenir le nouveau leader mondial de l'exploitation de nombreuses gemmes (saphirs, rubis et pierres semi-précieuses). Le sous-sol extrêmement riche a été très peu exploité et prouve régulièrement l'existence d'un potentiel géologique majeur au sein d'une multitude de gisement de tailles et d'intérêts économiques variables. Si ces derniers n'offrent généralement que très peu de perspectives pour les investisseurs internationaux, ils ont en revanche bien souvent les caractéristiques requises pour attirer les acteurs de la petite mine (illustration $n^{\circ} 3$ ).

Cette attractivité s'est renforcée depuis le début des années 1980, en raison de l'évolution des contextes sociaux, économiques et politiques de ces espaces. Les crises multiples et durables traversées par des pays comme Madagascar, la Tanzanie ou le Kenya offrent un substrat idéal au développement de ces activités artisanales et informelles (illustration 2). Depuis une vingtaine d'années, le déplacement du centre de gravité des productions de la rive Nord de l'océan indien vers l'Afrique orientale est une expression de l'ensemble de ces dynamiques. Chaque année, de nouveaux gisements apparaissent : les plus importants sont actuellement à Madagascar (Ilakaka, Andilamena...) et en Tanzanie (Arusha, Songea, Tunduru, Winza...) (illustration 4) mais tous les pays de la «ceinture du Mozambique» pourraient être touchés par des exploitations d'importance mondiale (Kenya, Somalie, Mozambique notamment) (Simonet, 2000). 
Illustration 4 - Les nouveaux espaces d'extraction des pierres précieuses (hors diamant) et des pierres fines en Afrique orientale

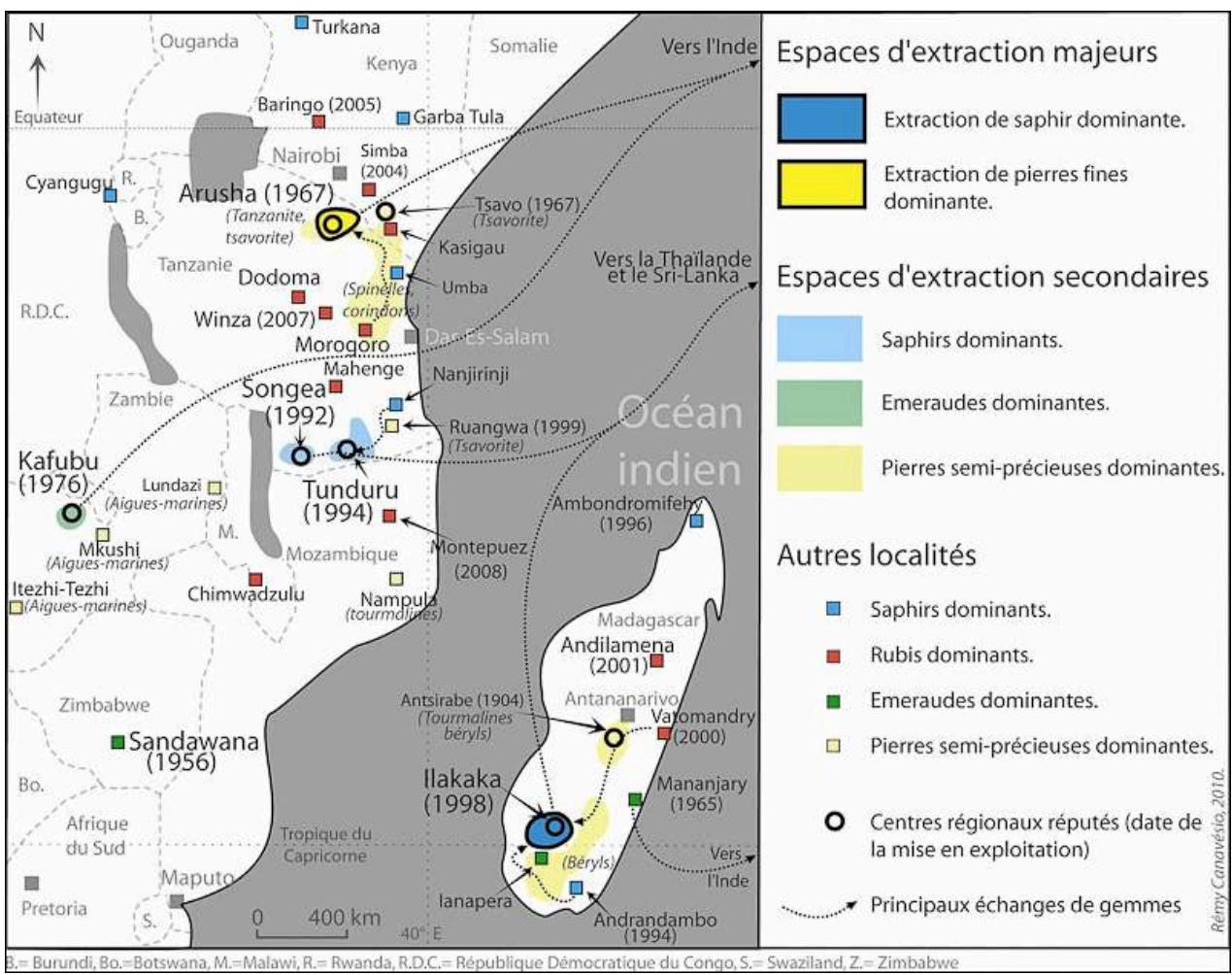

Les chiffres entre parenthèses correspondent à l'année au cours de laquelle l'exploitation de ces gisements a débuté.

Auteur : Remy Canavesio, 2010, modifié

Ainsi, alors que la géographie de l'extraction diamantifère africaine n'a quasiment pas évolué depuis l'ouverture des mines de la De Beers au Botswana au début des années 1980, la partie orientale du continent a en revanche été secouée par une multitude de ruées vers des espaces gemmifères nouveaux. Ces mouvements migratoires prennent parfois des dimensions exceptionnelles (souvent plusieurs milliers de personnes, parfois plusieurs dizaines de milliers) et sont à la fois source et conséquences des crises socio-spatiales qui ébranlent ces pays. Ils déstabilisent les sociétés locales, créent de nouvelles territorialités plus ou moins éphémères et projettent parfois très brutalement des espaces ruraux traditionnels dans des réseaux mondialisés plus ou moins criminels (Canavesio, 2010).

\section{Conclusion}

Alors que l'analyse par la législation minière peine à expliquer l'évolution de la mine artisanale, l'approche économique est efficace pour expliquer les transformations de la géographie mondiale des exploitations de saphirs et de rubis. Des évolutions complexes ont lieu tant au niveau de la demande (en raison de l'aspect très subjectif de la valeur des pierres) que de l'offre ( $\mathrm{du}$ fait de l'existence de deux types d'exploitations différentes, artisanales ou mécanisées). Ces dynamiques qui conditionnent directement l'évolution des espaces extractifs sont dans une large mesure spécifiques à ces 
gemmes : à la différence du diamant ou de l'or, ils ne sont pas stratégiques, et les acteurs industriels dans leur exploitation sont rares.

L'enrichissement des populations ne produit pas les mêmes effets sur la demande en produits de joaillerie d'une matrice historique et spatiale à l'autre. Si les pays dans lesquels les gemmes n'ont pas de significations particulières sont des marchés de choix pour la sphère diamantaire, les consommateurs appartenant à des ensembles culturels ayant une attraction millénaire pour les gemmes peuvent se distinguer par des comportements plus complexes.

Par ailleurs, si la hausse générale de la demande produit parfois des tensions au niveau de l'approvisionnement en certaines gemmes, l'enrichissement des populations dans certains pays producteurs peut aussi déboucher sur une réduction de la production provenant des exploitations artisanales de ces pays. Cela peut alors renforcer les tensions sur le marché international. Pour des pierres telles que les rubis et les saphirs, le dynamisme économique de l'Asie méridionale est en train de bouleverser en profondeur la géographie des espaces extractifs. Alors que la rive Nord de l'océan indien fournissait la majeure partie du marché mondial en saphirs, en rubis et en de nombreuses pierres semi-précieuses, l'amélioration des conditions de vie des habitants de plusieurs anciens pays producteurs asiatiques a provoqué le déplacement progressif du centre de gravité de l'activité extractive vers l'Afrique orientale. Après deux décennies d'activité de plus en plus soutenue dans ces nouveaux espaces gemmifères, c'est la question de la valorisation locale de ces ressources qui mérite d'être posée. L'exploitation des saphirs et des rubis s'inscrit-elle dans le paradigme de la malédiction des ressources naturelles (Rosser, 2006; Le Billon, 2005) pour les nouveaux pays producteurs? Cette question est devenue un enjeu majeur pour plusieurs régions d'Afrique orientale, en Tanzanie et à Madagascar notamment.

\section{BIBLIOGRAPHIE}

Brunet R., 2003. Le diamant, un monde en révolution.Paris, Belin, $414 \mathrm{p}$.

Campbell B., 2009. Mining in Africa. Regulation and development.Londres, Pluto Press, 284 p.

Canavesio R., 2010. Exploitation informelle des pierres précieuses et développement dans les nouveaux pays producteurs. Le cas des fronts pionniers d'Ilakaka à Madagascar. Thèse de Géographie, université Bordeaux III, sous la direction de Christian Bouquet, $502 \mathrm{p}$.

Canavesio R., 2006. Les filières « pierres précieuses » et « diamant » : la fin de deux trajectoires parallèles? Les Cahiers d'Outre Mer, $\mathrm{n}^{\circ}$ 236, p. 451 - 462

Canavesio R., 2004. La société malgache face à l'exploitation des saphirs d'Ilakaka. Mémoire de DEA, Université Bordeaux III, sous la direction de Christian Bouquet, $90 \mathrm{p}$.

De Geloes D’Elsloo R., Koffi P., Lataillade C., Monnet R., 2004. L'entreprise diamantaire dans la géopolitique africaine. Paris, L'Harmattan, $159 \mathrm{p}$. 
Di Méo G., Buléon P., 2005. L'espace social. Lecture géographique des sociétés. Paris, Armand Colin, $303 \mathrm{p}$.

Giuliani G., Chaussidon M., Schubnel H.J., Piat D., Rollion-Bard C., France-Lanord C., Giard D., De Narvaez D., Rondeau B., 2000. Oxygen isotopes and emerald trade routes since antiquity. Science, 28 janvier 2000, $n^{\circ} 287$, p. 631-633.

Hughes R-W., Ward F., 1997a. Burma: Heaven and hell in the hunt for jade. Asia Diamond, septembre-octobre 1997, vol. 1, nº 2, p. 42-53.

Hughes R-W., 1997b. Ruby and sapphire.U.S.A.: R.W.H. Publishing, 510 p.

Hughes R-W., 1996. Death of the Thai ruby. Jewelsiam, août-septembre 1996, vol. 7, nº 4, p. 100 105.

India Law Offices, 2008. Indian Jewellery Industry. 13 p., document pdf. Disponible sur : www.indialawoffices.com/industry_reports.php

Indian Embassy in Beijing. A guide for indian businesses. Gems and jewellery Industry in China. Pékin : Ambassade de l'Inde à Pékin, 2004. 103 p. En ligne, disponible au format pdf disponible sur : www.indianembassy.org.cn/pdf/Gems_Jewellery_Industry_in_China.pdf

KPMG., 2006. The Global Gems and Jewellery: Vision 2015: Transforming for Growth.

Lacroix A., 1992. Minéralogie de Madagascar, Tome I, Géologie minéralogie descriptive. Paris, Éditeur Augustin Challamel, $624 \mathrm{p}$.

Le Billon P., 2005. Geopolitics of ressource wars : Ressource dependence, governance and violence. London, Frank Cass, 288 p.

Misser F., Vallée O., 1997. Les gemmocraties : l'économie politique du diamant africain. Paris, Desclée de Brouwer, $243 \mathrm{p}$.

Pan A., 2008. China Gem and Jewellery Market Overview. Zeefer Consulting, Pékin, 148 p.

Rosser A., 2006. The political economy of the ressource curse: A literature survey. University of Sussex, $34 \mathrm{p}$.

Shor R., Weldon R., 2010. An Era of sweeping change in diamond and colored stone production and markets. Gems \& Gemology, vol. 46, n³, p. 166-187

Siboni J., 2007. Le luxe en Asie : Aujourd'hui, demain. ESSEC / Institut d'Etudes Politiques de Paris. Mémoire sous la direction de Jean-Marie Bouissou, 48 p. Document pdf disponible sur : www.siboni.net/resources/Luxe+Chine.pdf

Simonet C., 2000. Géologie des gisements de saphir et de rubis. L'exemple de la John Saul Ruby Mine. Mangare, Kenya. Thèse, Ecole de Gemmologie, Nantes, 350 p.

Smillie I, 2010. Blood on the stone. Greed, corrupion and war in the global diamond trade. New York, Anthem Press, 237 p.

Song Y., 2004. Brief Report on the Chinese Jewellery Industry. Shanghaï Flash, juillet 2004, ${ }^{\circ} 2$, $3 \mathrm{p}$.

Tsai J., 2008. La Chine et le luxe. Paris, Odile Jacob, 258 p. 


\section{NOTES}

1. La notion de gemme est relativement floue (Brunet, 2003) mais les apports sémantiques de la littérature anglo-saxonne et l'usage du terme dans le milieu des collecteurs de pierres utilisées en joaillerie permettent de considérer que les gemmes sont l'ensemble des éléments non organiques, transparents utilisés en joaillerie (Canavesio, 2010). On y trouve donc, lorsqu'ils sont transparents, les diamants, les pierres précieuses (saphirs, rubis, émeraudes) et un grand nombre de pierres fines (améthystes, aigues-marines...etc).

2. Dans le cadre de mon travail de terrain de DEA (Canavesio, 2004) et de doctorat (Canavesio, 2010) j'ai parcouru pendant près d'un an et demi (entre 2004 et 2008) plusieurs régions productrices de pierres précieuses de Madagascar (dont 6 mois dans la seule région d'Ilakaka). Une mission a également été menée dans la région de Chanthaburi en Thaïlande qui est un des principaux marchés mondiaux du commerce des saphirs et des rubis.

3. Les cartes présentées ici sont le produit de recherches de terrain et d'informations trouvées sur Internet. Dans ce domaine, le travail de Vincent Pardieu (membre du Gemological Institut of America à Bangkok) disponible sur le site Internet http://www.fieldgemology.org occupe une place importante.

4. La matrice historique et spatiale (Di Méo et Buléon, 2005) correspond à l'ensemble composé de faits et d'événement historiques dans un espace donné qui conduisent à la formation de phénomènes sociaux relativement homogènes. Ces matrices sont donc à l'origine de l'essentiel des particularités des sociétés (pratiques, modes de pensée...). Elles ont donc une influence majeure sur la consommation des gemmes.

5. Ce lien n'est pas systématique et, en Afrique de l'Est notamment, la découverte de pierres précieuses n'a pas provoqué d'intérêt particulier de la part des populations locales avant la seconde moitié du $20^{\mathrm{e}}$ siècle (Canavesio, 2010). Ce désintérêt est souvent mentionné par les colons européens (lire Alfred Lacroix, 1922, qui donne des précisions sur ce phénomène dans le cas malgache).

6. Les mines d'émeraude d'Habachtal en Autriche exploitées à l'époque romaine sont une des rares exceptions notables.

7. Ces variations peuvent s'expliquer de plusieurs manières : héritage colonial différent, opérations marketings inégales...

8. Les perles de culture sont également très appréciées en joaillerie. La part occupée par les perles dans la joaillerie nippone est tout à fait exceptionnelle. La valeur annuelle des bijoux sertis de perles s'élèverait à plus de 1 milliard US\$, soit 15 à $20 \%$ du marché global de la joaillerie.

9. La littérature ésotérique regorge d'ouvrages traitant de ce sujet. Dans la plupart d'entre-eux, des liens sont établis avec la culture, l'histoire et la tradition indienne. Les titres sont souvent évocateurs comme par exemple: Harish J., 1996. The Healing power of Gemstones: In Tantra, Ayurveda and Astrology. Inner Traditions Editions, Rochester, $240 \mathrm{p}$.

10. La comparaison de chiffres sur ce marché est trop aléatoire pour être menée avec fiabilité car il faut affronter le délicat problème des fraudes, du commerce informel, mais aussi l'appréciation très subjective de la qualité.

11. Cet aspect du marché se retrouve également en Inde : « Gold has traditionally been valued in India as a savings-and-investment vehicle and even today... (India Law Offices, 2008, p. 1).

12. Si des gisements sont connus dans le Yunan, l'essentiel de la production actuelle provient des gisements birmans de Mogaung, dans l'extrême Nord du pays (Hughes, 1997b). Dans le passé les gisements turkmènes ont également alimenté un commerce avec le marché chinois (Tsai, 2008).

13. Selon la Diamond Administration of China, la croissance des importations de diamants destinés au marché chinois se serait élevée à $30 \%$ entre 2008 et 2009.

14. Ces autres gemmes représentent $5 \%$ des importations officielles de pierres, mais ces chiffres doivent être maniés avec prudence car ils peuvent amplifier l'écart existant entre le diamant et 
les autres pierres. En effet, plus encore que le diamant, les autres gemmes sont soumises à de nombreux trafics. Les importations clandestines de jade sont par exemple massives à la frontière sino-birmane.

15. Amartya Sen considère que cette forme de crispation identitaire est en grande partie le résultat d'une évolution des partis politiques indiens actuels. Ces derniers, de plus en plus obsédés par l'indianité seraient les héritiers du discours tenu par les nationalistes élitistes de l'Inde coloniale. Par ailleurs, Sen ajoute que les éloges « exoticistes» formulés par de nombreux analystes occidentaux (et très largement relayés en Inde) depuis l'époque coloniale ont également entretenu et parfois accentué la singularité culturelle du monde indien.

16. Dans la région d'Ilakaka à Madagascar (le plus grand gisement de saphir au monde depuis 1998), l'exploitation artisanale représente plus de $90 \%$ de la production régionale (Canavesio, 2010).

17. L'estimation précise de la population concernée à l'échelle mondiale est délicate à réaliser. On peut néanmoins penser que, toutes gemmes confondues, ce sont pas loin de 2 millions de personne qui vivent directement de l'extraction artisanale, dont la moitié pour la seule exploitation du diamant.

18. L'aspect «faiblement » rémunérateur de ces activités informelles n'exclut pas des cas ponctuels d'enrichissement importants et imprévisibles.

19. Il existe de nombreux gisements connus de saphirs et de rubis en Australie ou dans l'Ouest des Etats-Unis, mais la plupart de ces gisements sont inexploités. En dehors du diamant, l'opale (exploitée en Australie) est le seul ornement utilisé en joaillerie faisant l'objet d'une activité extractive importante.

20. Les travaux menés pendant plusieurs années sur le terrain malgache ont montré que les évolutions de la législation minière n'avaient quasiment eu aucune influence sur la mine artisanale (Canavesio, 2010), alors que la mine formelle à forte concentration en capital est en revanche très réceptive à l'évolution des codes miniers. Or, celle-ci ne concerne guère les gemmes (hors diamant) (Campbell, 2009).

21. Alors que l'appropriation des gisements de diamant (comme ceux d'or ou de coltan) a été l'enjeu principal de nombreux conflits en Afrique (Angola, Sierra Leone, République Démocratique du Congo...), les autres gemmes n'ont jamais été à l'origine de conflits aussi importants. En effet, s'il est vrai qu'en Birmanie (rubis) et en Colombie (émeraudes), le contrôle des gemmes a pu être source de conflits armés, les pierres précieuses ont dans ces deux cas joué un rôle bien inférieur à celui des drogues (respectivement l'héroïne et la cocaïne).

22. Contrairement à l'exploitation de nombreux métaux tels que l'or, l'exploitation informelle des pierres précieuses ne fait appel à aucun produit chimique polluant. Les dégradations environnementales sont donc essentiellement liées à l'extraction, au transport et au stockage du minerais et du stérile. Dans ce domaine, la mine artisanale (qui opère souvent en milieu souterrain) a des effets bien inférieurs aux exploitations mécanisées qui - pour des raisons techniques - travaillent essentiellement sous la forme de mines géantes à ciel ouvert (Canavesio, 2010).

23. Le commerce illégal de diamant a été accusé de financer de nombreux conflits en Afrique au cours des années 1990 (Sierra Leone, Angola notamment).

24. Le traitement des gemmes permet d'améliorer l'apparence de ces dernières. Certains traitements (notamment thermiques) sont connus depuis des millénaires. De nouveaux traitements apparaissent néanmoins régulièrement (notamment le traitement au bérylium) et permettent à des pierres jusque-là invendables d'être commercialisées. 


\section{RÉSUMÉS}

L'évolution mondiale des activités extractives est de plus en plus dépendante de la demande des pays émergents. Les conséquences de la croissance de ces pays sur les exploitations de pierres précieuses sont complexes car le marché des gemmes a de nombreuses particularités. La demande est étroitement liée aux matrices socioculturelles de chaque pays. Par ailleurs, l'enrichissement des populations a également un impact sur la production de pierres telles que les saphirs ou les rubis. En effet, ces gemmes sont principalement extraites dans des exploitations informelles et cette activité est de moins en moins attractive pour une population dont le niveau de vie s'élève peu à peu. Dans les vastes gisements sri lankais et birmans, l'épuisement de la ressource est une autre menace. Finalement, si la croissance du marché du diamant est assurée par le Canada, la Russie et l'Australie, pour les autres gemmes, l'Afrique de l'Est est devenue le nouvel « Eldorado ». Dans ces pays, les contextes géologiques, économiques, politiques et sociaux sont très favorables au développement des exploitations artisanales de gemmes.

Extraction activities evolution is more and more dependent on the increase of demand in the emerging countries. The consequences of this growth on the gemstone mining activities are complex. The gemstone market is very special and the demand depends on the historic and cultural situation of every country. Moreover, for precious stones like rubby and sapphire, the supply coming from this emerging country is also affected by the social and economic changes. As the small scale mining is widely held in this activity, people that are becoming richer are less interested in that kind of job. In Sri Lanka and Burma, the depletion of many deposits is an other challenge. Finally, if new diamonds deposits of Canada, Russia and Australia are supplying the growth of the diamond market, Est Africa looks like the new "Eldorado " for the other gemstones. Geologic, economic, social and political context of these countries are perfectly adapted for the development of new gemstone small scall mining areas.

\section{INDEX}

Mots-clés : espaces extractifs, marchés, mine artisanale, pays émergents, pierres précieuses

Keywords : developing countries, extraction activities, gemstones, markets, small scale mining Thèmes : Sur le Champ - Sur le Terrain

\section{AUTEUR \\ REMY CANAVESIO}

Remy Canavesio est Docteur en géographie, ATER à l'Université de Polynésie française. remycanavesio@hotmail.fr 